\title{
Autofluorescence bronchoscopy: quantification of inter-patient variations of fluorescence intensity
}

\author{
Tanja Gabrecht • Blaise Lovisa • Huber van den Bergh • \\ Georges Wagnières
}

Received: 3 August 2007 / Accepted: 18 October 2007 /Published online: 30 November 2007

(C) Springer-Verlag London Limited 2007

\begin{abstract}
Autofluorescence (AF) from bronchial tissue is increasingly used for the endoscopic detection of early bronchial neoplasia. Several imaging systems are commercially available, all detecting the absolute or relative AF intensity and/or spectral contrasts between normal tissue and early neoplastic lesions. These devices have a high sensitivity for flat neoplasia, but the specificity remains limited. Variations in the AF intensity between individuals (interpatient variations) is considered one of the most limiting factors. In the clinical study presented here, we quantified those variations using a non-invasive optical reference positioned in situ during AF bronchoscopy. The inter-patient variations in intensity on the main carina were in the order of $25-30 \%$. The results of this study are quite useful for improving and defining the design of the optical features (dynamic range, physical sensitivity) of AF detection systems.
\end{abstract}

Keywords Autofluorescence bronchoscopy . Inter-patient variations · In vivo · Clinical · Imaging · Diagnostic autofluorescence endoscopy (DAFE) .

Dynamic range

\section{Introduction}

Autofluorescence bronchoscopy (AFB) using blue-violet excitation light is a powerful tool for the detection and location of early cancers and pre-cancers in the tracheo-

T. Gabrecht $\cdot$ B. Lovisa $\cdot$ H. van den Bergh $\cdot$ G. Wagnières $(\bowtie)$ Photomedicine Laboratory,

Swiss Federal Institute of Technology (EPFL),

Station 6, Building $\mathrm{CH}$,

1015 Lausanne, Switzerland

e-mail: georges.wagnieres@epfl.ch bronchial tract $[1,2]$. Its sensitivity exceeds that of conventional white-light bronchoscopy (WLB) by a factor of 2 [3-6]. AFB is based on the visualisation of the intensity and spectral contrasts of the autofluorescence (AF) of healthy bronchial mucosa and neoplastic or early cancerous lesions. Several AFB imaging systems are commercially available [3]. These systems use filtered arc lamps $[5,7,8]$ or laser sources [9] for the $\mathrm{AF}$ excitation, whereas $\mathrm{AF}$ imaging is performed with endoscopic colour cameras. One limitation of AFB that contributes to the limited specificity of this approach is due to the fluctuation of the AF intensity $[5,10,11]$. Spectrofluorometric in vivo studies have shown significant variations in the AF intensities of healthy bronchial mucosa among patients (interpatient variations) [12]. Those inter-patient variations can adversely affect the image quality in AFB and the learning curve of bronchoscopists. The unique complex morphology and anatomy of the tracheo-bronchia tree makes AFB imaging highly sensitive to inter-patient and intra-patient variations. One example of the inter-patient variations affecting AFB is the regular striped structure on the bronchial wall that is mainly present on the dorsal wall of the primary and secondary bronchi. The size and structure of these stripes can vary between patients, depending on anatomical variations and bronchial abnormality, mainly chronic inflammation and bronchitis. In AFB with the Richard Wolf diagnostic autofluorescence endoscopy (DAFE) system used in this study, the structures appear as white stripes alternating with dark red stripes, running along the longitudinal axis of the bronchi. The white stripes are formed by longitudinal bundles of elastic fibres situated just below the epithelium in the lamina propria of the bronchial wall. These bundles are less pronounced on the red stripes. Disruption and mutation of this stripe structure is an important diagnostic criterion in AFB. 
Knowledge of the inter-patient variations of the bronchial AF intensity is extremely important for the design and development of improved future AF imaging systems. However, the determination of the inter-patient variations with imaging systems is not easy. Indeed, clinical AFB imaging systems generally employ features such as camera gain control, frame integration, or adjustment of the shutter time to react to the dynamic imaging conditions during endoscopy. This allows the brightness of the visualised images to be adjusted to an optimal value, independent of the object's signal intensity. More specifically, the intensities of signals from tissue with low autofluorescence are amplified, while those from tissue with high autofluorescence are reduced. These adjustments are performed automatically by the system and can generally not be tracked either in real time, or retrospectively. In addition, the automatic white balancing procedure performed prior to each bronchoscopy in the white-light mode frequently influences the camera settings for the AFB mode. Quantification of the optical properties of tissues by an AFB system therefore demands the use of an optical/ fluorescence standard or reference that can be used at the endoscopic site during AF image recording. Such a reference, placed where it fills a small portion of the field of view, allows one to correct the tissue AF image for the settings of the AFB system at the time of recording and to compare AF signals from different endoscopic sites. The design and the characterisation of an endoscopic reference for use with the Richard Wolf DAFE system has recently been reported by Gabrecht et al. [13]. In that report we describe the detailed optical and spectral characterisation of this reference sample.

In this article we present a quantitative analysis of the inter-patient variations of AF intensity and reflectivity of endobronchial tissue. To our best knowledge, no such data obtained in vivo have previously been reported.

\section{Materials and methods}

The imaging system

AF imaging was performed with the DAFE system commercialised by Richard Wolf Endoscopes GmbH, Germany. Very briefly, the system consisted of an infrared (IR) filtered endoscopic xenon arc lamp light source and a filtered $3 \mathrm{CCD}$ endoscopic colour camera. A two-step flipflop filter holder containing a grid and a colour band pass filter allowed rapid changes between (1) violet AF excitation at $430 \mathrm{~nm} \pm 40 \mathrm{~nm}$ and (2) a conventional white light (WL) illumination (wavelength range $390 \mathrm{~nm}-$ $680 \mathrm{~nm}$ ). For AF detection, a variable amount of red light around $680 \mathrm{~nm}$ was added to the blue-violet $\mathrm{AF}$ excitation.
This light was detected to create a background image [14]. In this study the system was used with conventional Olympus bronchofibrescopes (Type BF30, Olympus, Japan). The power of the violet excitation light at the distal tip of the bronchofibrescope was $130 \mathrm{~mW}$. The endoscopic images were captured by the endoscopic camera and recorded on a digital video (DV) tape. A $475 \mathrm{~nm}$ high pass filter placed in the camera objective cut off all blue-violet excitation light. The spectral detection ranges for the three camera channels were $475 \mathrm{~nm}-490 \mathrm{~nm}$ (blue), $490 \mathrm{~nm}-580 \mathrm{~nm}$ (green), and $580 \mathrm{~nm}-650 \mathrm{~nm}$ (red).

The endoscopic optical reference

The endoscopic reference has been described in detail elsewhere [13]. Briefly, it was based on white opaque polymethyl methacrylate (PMMA) acrylic glass (Perspex Blanc Opal 1X20, Lucite Solutions, UK). A small cylinder ( $2 \mathrm{~mm}$ in diameter and $2 \mathrm{~mm}$ in length) was machined, provided with a central longitudinal drilling of $0.5 \mathrm{~mm}$ diameter and beaded onto a $0.5 \mathrm{~mm}$ surgical stainless steel guide wire (Unimed SA, Switzerland). The outer diameter of the cylinder was limited by the inner diameter of the bronchofibrescope working channel $(2.2 \mathrm{~mm})$. The optical properties of this PMMA resemble those of human bronchial tissues. More precisely, the fluorescence emission of the material and its reflectance in the red part of the spectrum are similar to those of human bronchial mucosa in the spectral conditions of the DAFE system. A detailed description of the optical properties can be found in [13]. An adjustable marker positioned at the proximal end of the guide wire ensured a constant distance of $7 \mathrm{~mm}$ between the bronchoscope tip and the reference during measurements. The position of the marker was adjusted prior to each session of bronchoscopy.

\section{Patients}

All 11 patients (five female and six male) involved in this study underwent diagnostic DAFE bronchoscopy in the ENT department of Centre Hospitalier Universitaire Vaudois (CHUV) the University Hospital in Lausanne, Switzerland. The mean age was $59 \pm 5.3$ years (range 49 to 67 years). All patients were considered to be at high risk for head and neck or bronchial cancer, with nine patients being longtime smokers and two being non-smokers. The bronchoscopies were carried out under general anaesthesia using a $7.5 \mathrm{CH}$ bronchoscope for intubation. The measurements were performed close to the optically healthy, i.e. tissues not suspicious under WL and AF bronchoscopy, main carina. The optical exclusion criteria were: areas of suspicion under $\mathrm{WL}$ or AF bronchoscopy in the proximity of the carina, and bleeding or mucous at the bronchoscopic site. The AFB 
study was approved by the local ethics committee. All patients were asked to give informed consent prior to being enrolled in the study.

\section{Clinical protocol}

Following examination of the entire bronchial tree with both AF and conventional WL bronchoscopy, the flexible bronchofibrescope was positioned in front of the main carina. The visual aspect of the site was assessed for normality by both AFB and WLB. Then, the endoscopic reference was inserted into the working channel of the bronchofibrescope and positioned beside the main carina. As reported by Gabrecht et al.[13], the reference was positioned at a minimal distance from the bronchial wall to avoid a modification of the reference signal by the spatial irradiance coming from the bronchial wall. Images of the reference positioned close to the main carina were recorded in the AFB mode. The whole procedure took less than 2 min and did not interfere with the bronchoscopy. All AF bronchoscopies were performed according to the guidelines approved by the ethics committee of CHUV University Hospital.

\section{Image analysis}

AF images showing the reference close to the main carina were digitised in 24-bit red-green-blue (RGB) mode from the DV recordings using the IEEE1394 interface of the DV recorder and a portable personal computer (PC). Image analysis was performed with Adobe Photoshop 7.0 software (Adobe Systems, San Jose, CA, USA). In each image four areas were selected and analysed for the values of their red (R) and green (G) intensity levels: (1) the face side of the reference, i.e. the surface of the cylinder facing the observer, (2) a tissue area on the main carina, (3) and (4) an area on a red and a white stripe, respectively. The selected area on the reference typically included 1,000-1,500 pixels, corresponding to a surface of $2 \mathrm{~mm}^{2}$. A corresponding area was selected on the tissue. The position of the reference close to the carina and the selection of the analysed areas are illustrated in Fig. 1. Saturated image areas as well as those showing specular reflections and surface abnormalities (secretion, airway crypts, etc.) were excluded from the analysis. All computed values of $\mathrm{R}$ and $\mathrm{G}$ intensity levels were corrected for the image background and the gamma factor of the camera system.

In order to quantify the inter-patient variations in intensity of the $\mathrm{AF}$, we computed three green intensity ratios between (1) the reference area and the area on the carina, (2) the reference area and the area on the red stripes, and (3) the reference area and the area on the white stripes. In the following sections, these ratios will be referred to as

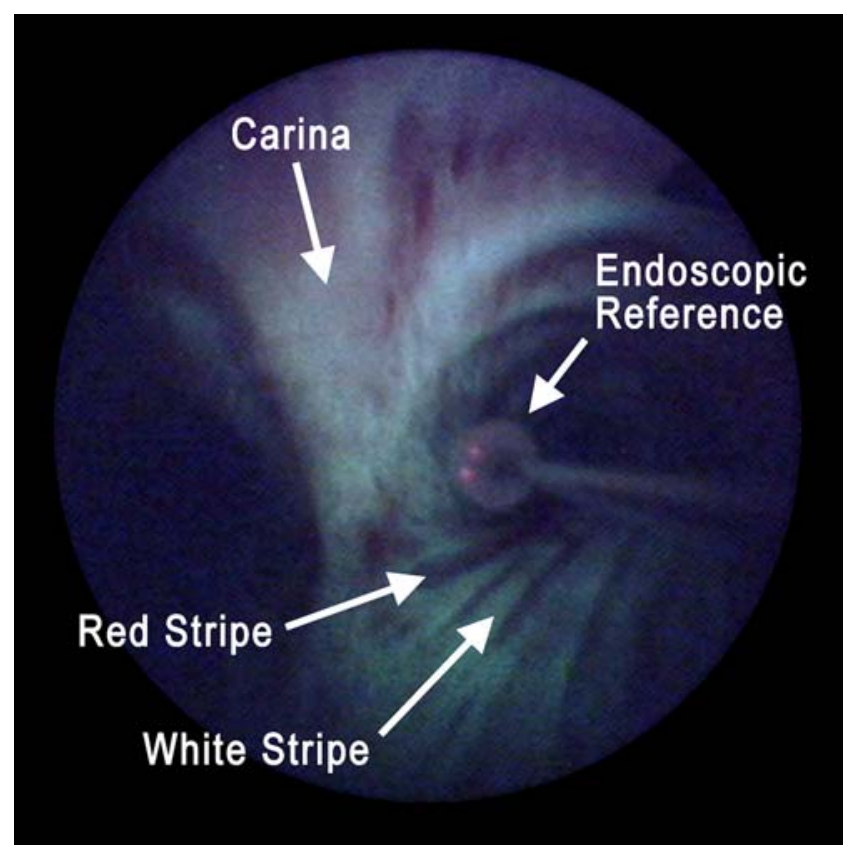

Fig. 1 DAFE image of the main carina, showing the endoscopic reference. The brightness and the chromatic aspect of the reference are similar to those of the bronchial wall. The striped structures on the dorsal wall (bottom) of the primary bronchi are clearly visible (A colour version of the image can be found in the online publication of this article)

the $\left(\mathrm{G}_{\text {ref }} / \mathrm{G}_{\text {tis }}\right)$ ratios. The corresponding $\left(\mathrm{R}_{\text {ref }} / \mathrm{R}_{\text {tis }}\right)$ ratios were computed accordingly.

Moreover, we computed the so-called normalised redto-green ratio $\left((\mathrm{R} / \mathrm{G})_{\text {norm }}\right)$, which is defined as the ratio between the red and the green intensity levels of the reference $\left(\mathrm{R}_{\mathrm{ref}} / \mathrm{G}_{\mathrm{ref}}\right)$ divided by the ratio between the red and the green intensity levels from the bronchial wall $\left(\mathrm{R}_{\mathrm{tis}} / \mathrm{G}_{\mathrm{tis}}\right)$. In other words,

$$
\left(\frac{\mathrm{R}}{\mathrm{G}}\right)_{\text {norm }}=\left(\frac{\mathrm{R}_{\text {ref }}}{\mathrm{G}_{\text {ref }}}\right) \div\left(\frac{\mathrm{R}_{\text {tis }}}{\mathrm{G}_{\text {tis }}}\right)
$$

The $(\mathrm{R} / \mathrm{G})_{\text {norm }}$ ratio is of interest, as it is a monotonic function of the chromatic aspects of the image obtained with the DAFE system. It should be kept in mind that the green signal results from the tissue AF only, while the red signal is composed of both the tissue $\mathrm{AF}$ and the backscattering signal.

Typically, three different AF images per patient were digitised, analysed, and averaged. Our image analysis procedure was based on the selection of zones within an endoscopic image. To determine at which point the values of the computed $\left(\mathrm{G}_{\mathrm{ref}} / \mathrm{G}_{\mathrm{tis}}\right)$, $\left(\mathrm{R}_{\mathrm{ref}} / \mathrm{R}_{\text {tis }}\right)$ and $(\mathrm{R} / \mathrm{G})_{\text {norm }}$ ratios depend on the selections performed, the standard deviations were deduced from these measurements.

The error of the image analysis was deduced from the variation of the intensity levels computed from multiple tissue areas selected independently on the same bronchoscopic site. 
Fig. 2 Green intensity ratios of the reference and the bronchial tissues measured in 11 patients. The ratios are presented for the carina, the red stripes and the white stripes. The horizontal lines depict the mean ratios for the carina (dashed line), the red stripes (continuous line) and the white stripes (large dashed line). The error bars depict the standard deviations

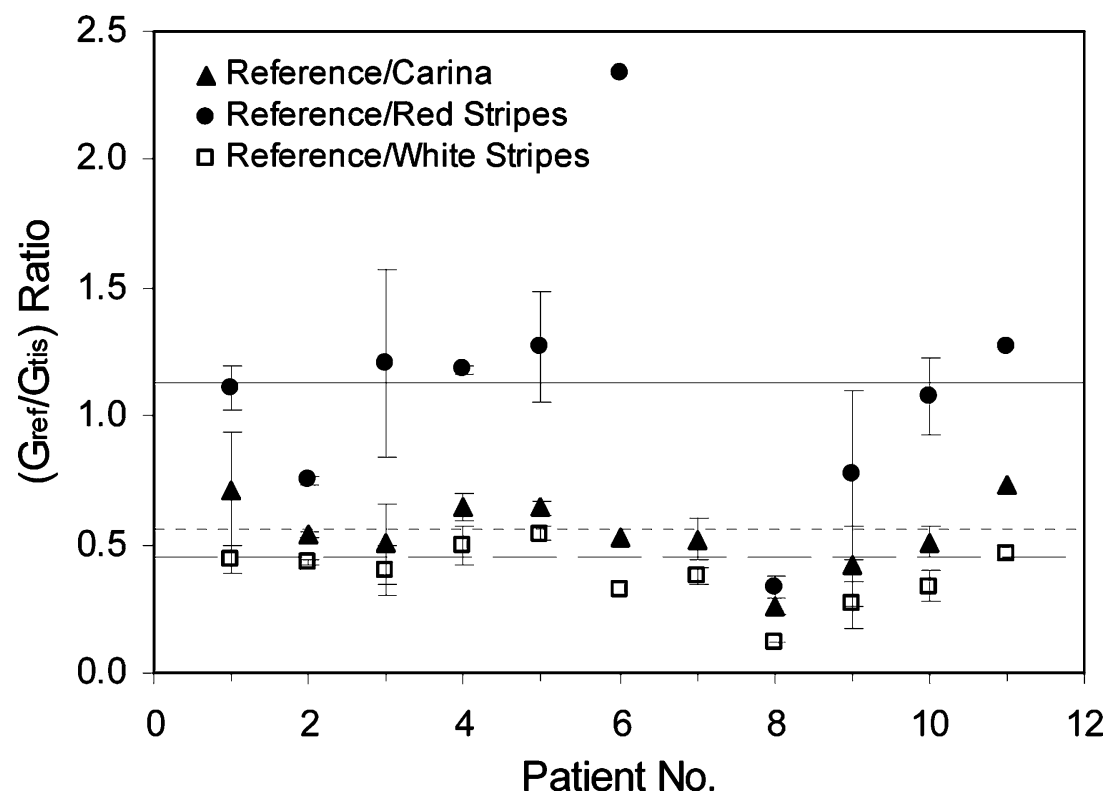

\section{Results}

The results from the image analysis are shown in Figs. 2, 3 and 4 for $\left(\mathrm{G}_{\mathrm{ref}} / \mathrm{G}_{\text {tis }}\right),\left(\mathrm{R}_{\mathrm{ref}} / \mathrm{R}_{\text {tis }}\right)$ and $(\mathrm{R} / \mathrm{G})_{\text {norm }}$, respectively.

Figure 2 shows the $\left(\mathrm{G}_{\text {ref }} / \mathrm{G}_{\text {tis }}\right)$ ratios between the reference and (1) the carina (triangles), (2) the red stripes (diamonds) and (3) the white stripes (squares) for all 11 patients. Each point represents the mean of three ratios computed from the three different images of a given patient, and the error bars depict the standard deviations. The mean $\left(\mathrm{G}_{\text {ref }} / \mathrm{G}_{\text {tis }}\right)$ ratios between the reference and the tissue over all cases are $0.55 \pm 0.14$ for the carina, $1.13 \pm 0.52$ for the red stripes, and $0.38 \pm 0.12$ for the white stripes. The mean ratios for each zone are depicted as dashed horizontal lines in Fig. 2. The corresponding $\left(\mathrm{R}_{\text {ref }} / \mathrm{R}_{\text {tis }}\right)$ ratios are shown in Fig. 3. Depiction of the ratios and mean values are the same as in Fig. 2. The mean $\left(\mathrm{R}_{\text {ref }} / \mathrm{R}_{\text {tis }}\right)$ ratios between the reference and the tissue are $0.77 \pm 0.25$ for the carina, $1.27 \pm$ 0.68 for the red stripes, and $0.63 \pm 0.2$ for the white stripes. It should be noted that the $\left(\mathrm{G}_{\mathrm{ref}} / \mathrm{G}_{\text {tis }}\right)$ and $\left(\mathrm{R}_{\mathrm{ref}} / \mathrm{R}_{\mathrm{tis}}\right)$ ratios between the reference and the carina, as well as those between the reference and the white stripes, are all smaller than 1 , while most of the corresponding ratios for the red stripes exceed 1 .

The $(\mathrm{R} / \mathrm{G})_{\text {norm }}$ ratios for the carina are shown in Fig. 4 for all 11 patients. As in Figs. 2 and 3, each datum point
Fig. 3 Red intensity ratios of the reference and the bronchial tissues measured in 11 patients. The ratios are presented for the carina, the red stripes and the white stripes. The depiction is the same as in Fig. 2. The error bars depict the standard deviations

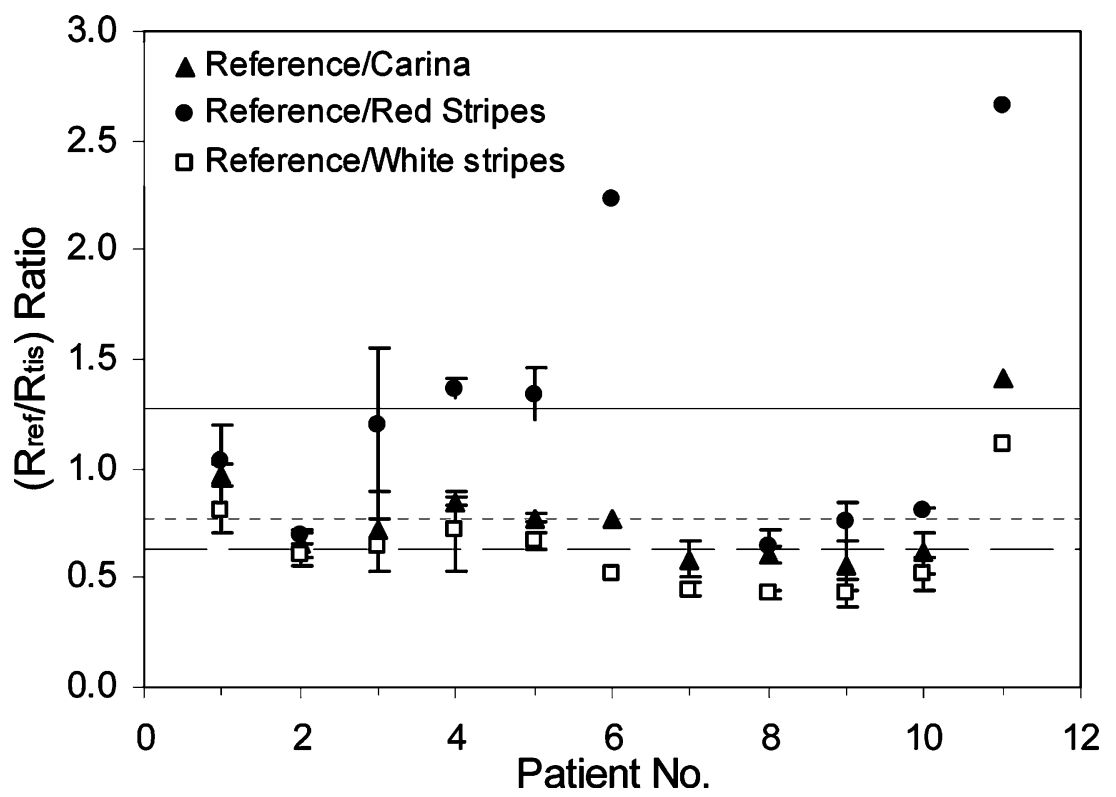


Fig. 4 Normalised (R/G) intensity ratios between the reference and the bronchial carina, the red stripes, and the white stripes, measured in 11 patients. The error bars depict the standard deviations

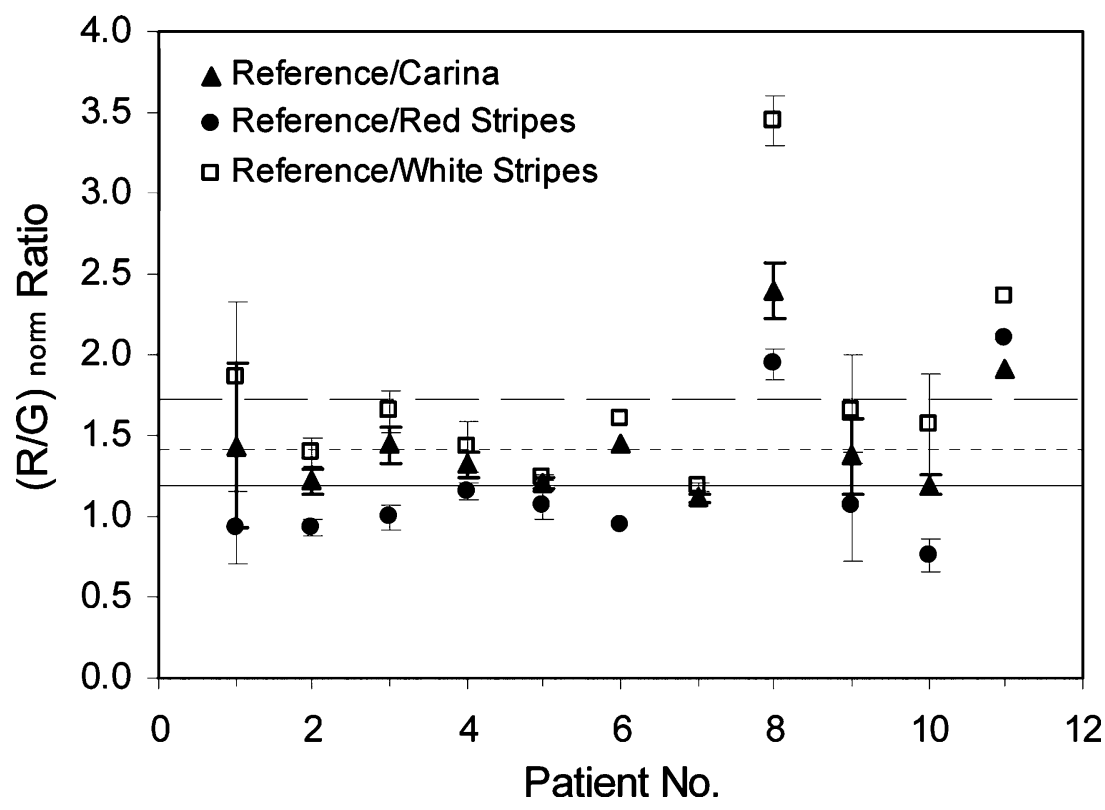

represents the mean of typically three ratios, and the error bars depict the standard deviations. The dotted horizontal line shows the mean $(\mathrm{R} / \mathrm{G})_{\text {norm }}$ ratio. The mean $(\mathrm{R} / \mathrm{G})_{\text {norm }}$ ratios were $1.46 \pm 0.38(26 \%)$ for the carina, $1.19 \pm 0.45$ $(38 \%)$ for the red tracks and $1.76+-0.65(37 \%)$ for the white tracks.

The errors resulting from our image analysis procedure were approximately $15 \%$ for the $\left(\mathrm{G}_{\text {ref }} / \mathrm{G}_{\text {tis }}\right)$ and $\left(\mathrm{R}_{\mathrm{ref}} / \mathrm{R}_{\mathrm{tis}}\right)$ intensity level ratios and approximately $11 \%$ for the $(\mathrm{R} / \mathrm{G})_{\text {norm }}$ ratios.

\section{Discussion}

The autofluorescence spectra of human bronchial tissue have been studied by several groups [12, 15-17]. However, the inter-patient variations of the AF intensity and spectral composition have hardly been studied quantitatively in vivo. Zellweger and co-workers [12] reported significant inter-patient variations of approximately $50 \%$ within the $67 \%$ confidence interval using $405 \mathrm{~nm}$ as excitation wavelength. Compared to those values, the inter-patient variations found in our study were surprisingly small. The inter-patient variations measured on the healthy carina were approximately $25 \%$ for the AF intensity in the green part of the spectrum $(490 \mathrm{~nm}-580 \mathrm{~nm})$ and only slightly higher $(32 \%)$ in the red part of the spectrum. The relative ratio between the green and the red spectral parts of the AF varied by about $26 \%$. Those values are nearly twice that of the error related to the image analysis method in this study. The differences between the inter-patient variations found by Zellweger et al. and those presented in this report most likely result from the measurement geometry used in the two studies. Zellweger et al. performed spectrofluorometric measurements using a fibre bundle and a spacer to keep a tissue-bundle distance of $3.5 \mathrm{~mm}$ [18]. This set-up offers a relatively small probing area for a given measurement. Consequently, local non-homogeneities of the tissue AF in the millimetre range may dramatically influence the $\mathrm{AF}$ signals measured from different spots in a given patient or between patients. Moreover, the AF intensities measured with this set-up are highly sensitive to slight changes in the tissue-probe distance. Our results were obtained using a broad field imaging technique. This measurement geometry is much less sensitive to non-homogeneities of the tissue $\mathrm{AF}$ in the millimetric range and to slight variations in the tissue-probe distance, since the tissue-endoscope distance is approximately $10 \mathrm{~mm}$.

In this context, it is worth noting that the inter-patient variations observed in this imaging study were significantly higher $(31 \%$ and $46 \%)$ on the white and red stripes, respectively, of the dorsal bronchial wall than on the carina $(25 \%)$. As already pointed out in the introduction, the white stripes are formed by dense bundles of highly fluorescent elastic fibres that are closely juxtaposed to the epithelium. On the red stripes the fibre bundles are missing, resulting in a much lower AF intensity. Thus, the signal from the red stripes is dominated by the red backscattered light. The higher variations observed on the stripes are most likely due to the small size of both types of stripes (and consequently the small size of the analysed areas) making image analysis more sensitive to heterogeneities in the tissue's emission.

Though the inter-patient variations in the bronchial tissue AF observed in the this study were small, it is known from clinical practice with AFB that autofluorescence images obtained from different patients can show 
clear differences in their chromatic compositions and intensities [3]. This is likely to be partly due to variations in the system's performance from one bronchoscopy to another, including variations of the excitation light intensity at the tip of the bronchoscope optics, the transmission quality of the imaging optics, and colour settings of the camera display unit, among others. Calibration charts or references used prior to the bronchoscopic examination can reduce these instrumental variations. Surprisingly, virtually no such test reference samples are provided by manufacturers producing AFB systems.

An additional approach to level out inter-patient variations in AFB is the use of automatic colour balance (ACB) procedures during bronchoscopy. Likewise the automatic white balance employed by nearly all conventional endoscopic imaging systems, the ACB will adjust the gains of the individual RGB colour channels to obtain a preset RGB signal. Performed on healthy tissue, this will allow compensation for potential colour shifts in the AFB image due to the inter-patient variations of the tissue AF.

This pioneering clinical study conducted with the endoscopic reference shows that its optical and fluorescence properties differ slightly from those of human bronchial tissue. As reported by Gabrecht et al. [13], the diffuse reflectance of the endoscopic reference is slightly higher than that of human bronchial mucosa at $675 \mathrm{~nm}$. However, the AF emission spectrum of the reference was found to be slightly blue shifted relative to the tissue AF, resulting in a higher $R / G$ ratio for the reference. This is confirmed by the fact that all $(\mathrm{R} / \mathrm{G})_{\text {norm }}$ ratios computed for the carina in the study detailed here were greater than 1 . Moreover, the mean $\left(\mathrm{G}_{\mathrm{ref}} / \mathrm{G}_{\text {tis }}\right)$ ratios computed for the main carina were 0.55 . This indicates that the $\mathrm{AF}$ intensity emitted by the endoscopic reference in the green spectral part, i.e. between $490 \mathrm{~nm}$ and $580 \mathrm{~nm}$, was approximately half the AF intensity typically emitted by healthy bronchial tissues. Although the AF of the tissue and that of the reference sample were about the same, this relatively low AF of the Perspex material was not ideal, in the sense that the reference emission was more affected by the environmental space irradiance induced by the tissues. The most reliable results will, therefore, be obtained with a brighter reference. Thus, for further clinical studies, the choice of a more fluorescent reference material than the one used in our study should be considered. It should be kept in mind that the procurement of material presenting optical and spectral properties similar to those of the bronchial wall, while being suitable for this clinical use, is very challenging. In addition, it is crucial to prevent saturation of the image, which will occur if the reference is too bright.

Apart from the inter-patient variations, the differences in the AF intensity and spectral composition within the bronchial tree of a given patient (the so-called intra-patient variations) are also very likely to influence the performance of AFB. Intra-patient variations result from morphological changes of the bronchial wall observed between the lower and higher level bronchi $[3,19]$. These changes are, at least in part, due to variations in the epithelium thickness and complexity, the number and distribution of specific cells and the spatial distribution of smooth muscle and cartilage in the bronchial wall. A clinical study by our group, aiming to quantify these intra-patient variations using the endoscopic reference at different levels of the tracheo-bronchial tree, is currently in progress.

\section{Conclusions}

In this article we detailed the use of an endoscopic reference for the determination and quantification of interpatient variations in the $\mathrm{AF}$ and reflectance of human bronchial tissue, using AFB imaging. The inter-patient variations in the bronchial $\mathrm{AF}$ intensity and spectral composition found on the carina with the AFB imaging system are small and, therefore, probably do not significantly affect the performance of AFB. The endoscopic reference described here presents a rapid and minimally invasive method to determine and quantify the intensity and spectral composition of bronchial AF during AFB.

\section{References}

1. Häußinger K, Stanzel F, Kohlhäufl M, Becker H, Herth F, Kreuzer A, Schmidt B, Strausz J, Cavaliere S, Müller K-M, Huber R-M, Pichlmeier U, Bolliger CT (2005) Autofluorescence bronchoscopy with white light bronchoscopy compared with white light bronchoscopy alone for the detection of precancerous lesions: a European randomised controlled multicentre trial. Thorax 60: 496-503

2. Lam S, MacAulay C, leRiche JC, Palcic B (2000) Detection and localization of early lung cancer by fluorescence bronchoscopy. Cancer Suppl 89:2468-2473

3. Wagnières G, McWilliams A, Lam S (2003) Lung cancer imaging with fluorescence endoscopy. In: Myceck M-A, Pogue BW (eds) Handbook of biomedical fluorescence, Marcel Dekker, New York, pp 361-396

4. Lam S, Kennedy T, Unger M (1998) Localization of bronchial intraepithelial neoplastic lesions by fluorescence bronchoscopy. Chest 113:696-702

5. Goujon D, Zellweger M, Radu A, Grosjean P, Weber B-C, van den Bergh H, Monnier P, Wagnières G (2003) In vivo autofluorescence imaging of early cancers in the human tracheobronchial tree with a spectrally optimized system. J Biomed Opt 8:17-25

6. Pierard P, Martin B, Verdebout J-M, Faber J, Richez M, Sculier J-P, Ninane V (2001) Fluorescence bronchoscopy in high-risk patients - comparison of LIFE and Pentay systems. J Bronchology 8:254-259

7. Leonhard M (1999) New incoherent autofluorescence/fluorescence system for early detection of lung cancer. Diagn Ther Endosc 5:71-75 
8. Häußinger K, Stanzel F, Huber RM, Pichler J, Stepp H (1999) Autofluorescence detection of bronchial tumors with the D-Light/ AF. Diagn Ther Endosc 5:105-112

9. Ikeda N, Honda H, Hayashi A, Usuda J, Kato Y, Tsuboi M, Ohira T, Hirano T, Kato H, Serizawa H, Aoki Y (2006) Early detection of bronchial lesions using newly developed videoendoscopybased autofluorescence bronchoscopy. Lung Cancer 52:21-27

10. Hirsch FR, Prindiville SA, Miller YE, Franklin WA, Dempsey EC, Murphy JR, Bunn Jr PA, Kennedy TC (2001) Fluorescence versus white-light bronchoscopy for detection of preneoplasic lesions: a randomized study. J Natl Cancer Inst 93:1385-1391

11. Kennedy TC, Lam S, Hirsch FR (2001) Review of recent advances in fluorescence bronchoscopy in early localization of central airway lung cancer. Oncologist 6:257-262

12. Zellweger M, Goujon D, Conde R, Forrer M, van den Bergh H, Wagnières G (2001) Absolute autofluorescence spectra of human healthy, metaplastic, and early cancerous bronchial tissue in vivo. Appl Opt 40:3784-3791

13. Gabrecht T, Lovisa B, Borle F, Wagnieres G (2007) Design of an endoscopic optical reference to be used for autofluorescence bronchoscopy with a commercially available diagnostic autofluorescence endoscopy (DAFE) system. Phys Med Biol 52:N163-N171
14. Gabrecht T, Glanzmann T, Freitag L, Weber B-C, van den Bergh H, Wagnières G (2007) Optimised autofluorescence bronchoscopy using additional backscattered red light. J Biomed Opt (in press)

15. Alfano R, Tata D, Cordero J, Tomashefsky P, Longo F, Alfano M (1984) Laser induced fluorescence spectroscopy from native cancerous and normal tissue. IEEE J Quantum Electron 20:1507-1511

16. Alfano R, Tang G, Pradhan A, Lam W, Choy D, Opher E (1987) Fluorescence spectra from cancerous and normal human breast and lung tissues. IEEE J Quantum Electron 23:1806-1811

17. Qu J, MacAulay C, Lam S, Palcic B (1995) Laser-induced fluorescence spectroscopy at endoscopy: tissue optics, Monte Carlo modeling and in vivo measurements. Opt Eng 34: 3334-3343

18. Zellweger M, Grosjean P, Goujon D, Monnier P, van den Bergh $H$, Wagnières G (2001) In vivo autofluorescence spectroscopy of human bronchial tissue to optimize the detection and imaging of early cancers. J Biomed Opt 6:41-51

19. Gabrecht T, Andrejevic-Blant $S$, Wagnières G (2007) Blue-violet excited autofluorescence spectroscopy and imaging of normal and cancerous human bronchial tissue after formalin fixation. Photochem Photobiol 83:450-458 